\title{
Rare Case of Wernicke Encephalopathy with Initial Negative Diffusion-Weighted Imaging
}

\author{
Halil Onder $M D^{1}$ iD, Serdar Kirmizi $M D^{2}$ iD
}

${ }^{1}$ Department of Neurology, Yozgat City Hospital, Turkey

${ }^{2}$ Department of Gastric Surgery, Yozgat City Hospital, Turkey

Date of submission: $26^{\text {th }}$ April 2021

Date of acceptance: $4^{\text {th }}$ November 2021

Date of publication: $1^{\text {st }}$ December 2021

\section{Abstract}

In this report, we present a rare patient with Wernicke encephalopathy (WE) in whom the initial magnetic resonance imaging (MRI) was normal. However, cranial MRI, performed two weeks later, showed lesions compatible with WE. Via the presentation of this patient, we discuss the need for future studies of larger cases including the temporal evaluation of the MRI characteristics of Wernicke encephalopathy.

Key words: Diagnosis, Diffusion-weighted imaging, Wernicke encephalopathy.

\section{Introduction}

W ernicke encephalopathy (WE) is a critical form of metabolic encephalopathy resulting from acute thiamine deficiency and can be reversible with timely initiation of treatment. The diagnosis is based on the clinical findings and neuroimaging parameters. ${ }^{1}$ However, the temporal change of the neuroimaging findings and the reliability of MRI-based diagnosis have not been investigated in detail up to date. Herein, we will illustrate a remarkable case of WE with initial negative MRI results. However, the subsequent MRI showed lesions supporting the WE and the intravenous thiamine was initiated which provided dramatic improvement in his clinic.

\section{Case report}

A 57-year-old male was admitted to our emergency with complaints of severe nausea and vomiting which

Access this article online
Website: https://www.nepjol.info/index.php/NJN
DOI: https://doi.org/10.3126/njn.v18i4.36629
HOW TO CITE
Onder H, Kirmizi S. Rare Case of Wernicke Encephalopathy
with Initial Negative Diffusion-Weighted Imaging. NJNS.
2021;18(4):72-4.

Address for correspondence:

Dr. Halil Onder

Yozgat City Hospital Center,

Yozgat, Turkey.

E-mail: halilnder@yahoo.com

Phone: +9005376836864

Copyright (C) 2021 Nepalese Society of Neurosurgeons (NESON)

ISSN: 1813-1948 (Print), 1813-1956 (Online)

This work is licensed under a Creative Commons Attribution-Non Commercial 4.0 International License. had started over the last few weeks and progressed. His medical history revealed that the patient had undergone a Whipple procedure due to clinical suspicion of duodenal malignancy, however, the pathological investigations showed duodenal diverticula. The surgery had been performed in another medical center and further data regarding the indication for that surgery was unavailable. At admission to our emergency service, the patient had mild difficulty in cooperation with the examination; and orientation to time was disturbed. Cranial nerve examinations revealed that the patient had difficulty in vertical and horizontal eye movements. Other cranial nerve investigations were within normal limits. Motor and sensory examinations were normal. However, he had significant truncal ataxia such that he could only mobilize a very short distance without support. The patient had no history of alcohol abuse or exposure to a chemical. Vital signs were within normal ranges. Laboratory investigations including hemogram, biochemistry, TSH, and folic acid were within normal limits. Serum B12 level was moderately reduced (B12: $136 \mathrm{pg} / \mathrm{mL}$ ). Due to his medical problems, to avoid longer scan time, rather than an extensive MRI protocol, cranial diffusion-weighted imaging (DWI) was performed which revealed normal findings. (Figure 1)

Due to the severe vomiting and nausea, his oral intake was severely disturbed over the last week. The endoscopic investigation revealed an obstruction in the gastroenterostomy anastomosis. Laparoscopic revision of the gastroenterostomy anastomosis was performed. After the surgery, oral intake has significantly improved. However, his cognitive function further deteriorated in the following course, such that the patient was noncooperative and disorientated. Besides, the patient could not be mobilized without support due to severe truncal ataxia. Cranial MRI was repeated two weeks later which showed hyperintensity in T2-weighted imaging and DWI 


\section{Wernicke Encephalopathy with Initial Negative DWI Images}

in bilateral medial thalami and periventricular region of the third ventricle. (Figure 2)

Taken together, the diagnosis of Wernicke encephalopathy (WE) was established and intravenous thiamine was initiated (thiamine $3 \times 200 \mathrm{mg}$ for 3 days and 1x200 mg daily in the following 5 days) which provided a significant recovery in the clinic. In the second week of the treatment, the patient was fully cooperative and orientated. Mini-mental state examination score was 26 points. Truncal ataxia was significantly improved such that he could easily mobilize without support.

\section{Discussion}

Wernicke encephalopathy is a life-threatening but reversible syndrome resulting from acute thiamine deficiency that is frequently overlooked and underdiagnosed. ${ }^{2}$ It is a medical emergency and early diagnosis of this entity is critical as it can lead to severe neurologic deficits like Korsakoff psychosis and even death if appropriate treatment is not administered. However, it is reported that WE diagnosis may be missed in up to $75-80 \%$ of patients with alcoholism and AIDS. ${ }^{1}$ Gastrointestinal surgery and prolonged vomiting are also crucial medical conditions that may impair the correct absorption of a sufficient amount of thiamine potentially leading to WE. ${ }^{3,4}$ In our patient, the clinical presentation and the medical history of gastrointestinal surgery and vomiting also suggested the WE. However, the initial DWI did not show any finding supporting the diagnosis. Remarkably, MRI that was repeated two weeks later confirmed the diagnosis of WE. We think that the neuroimaging data of this patient may give crucial perspectives for further deliberations.

The clinical diagnosis of WE is based on the classical triad consisting of ocular signs, altered consciousness, and ataxia that is initially described by Wernicke in his original

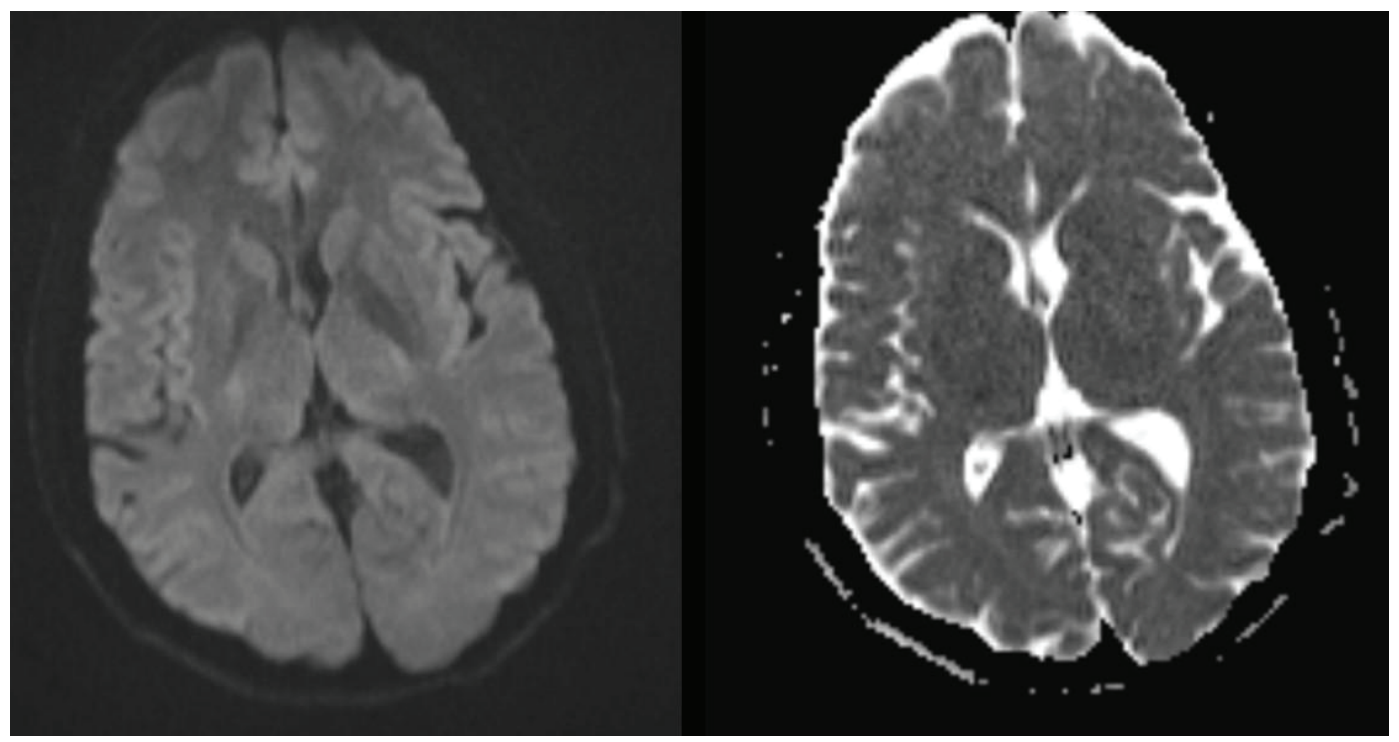

Figure 1: The diffusion-weighted imaging at admission showing a normal appearance.

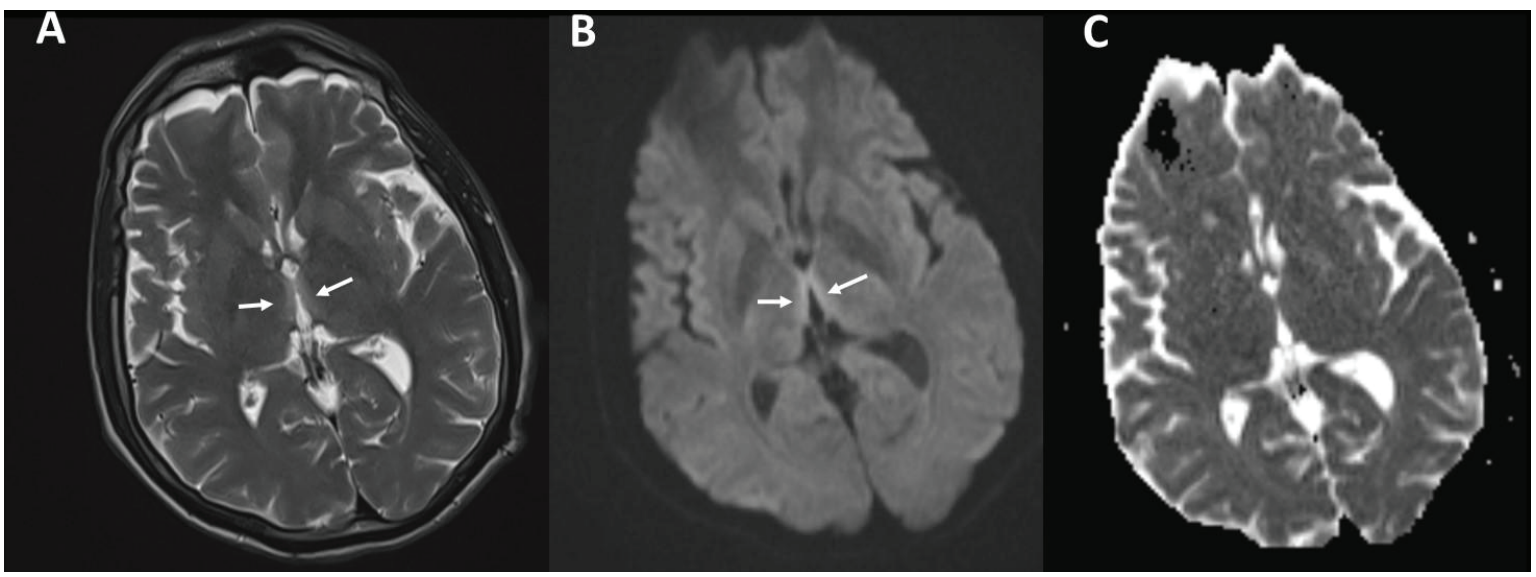

Figure 2: The cranial MRI, performed two weeks after admission, shows hyperintensity in T2-weighted imaging and DWI in bilateral medial thalami and periventricular region of the third ventricle (A: T-2 weighted imaging, $B$ : DWI sequence, $C$ : ADC sequence). 
article. ${ }^{5}$ However, subsequent studies have revealed that this triad occurs in only $16-38 \%$ of all patients with WE. ${ }^{6,7}$ Thereafter, MRI has been emphasized as a critical and useful tool for the diagnosis of WE. ${ }^{4,6}$ Reversible cytotoxic edema is considered the most distinctive lesion of WE, and it is easily shown on conventional MRI. ${ }^{4}$ Regions including bilateral thalami, mammillary bodies, tectal plate, and periaqueductal area are reported as typical lesion sites in WE. ${ }^{8}$ However, the absence of MR signalintensity alterations does not exclude the diagnosis of WE. ${ }^{8}$ The remarkable point was that we performed only DWI initially which might be the reason for the negative result. DWI is a sensitive tool to show cytotoxic edema that is considered to play a major role in the mechanisms of WE and it is surely a valuable additional imaging sequence for the diagnosis of WE. ${ }^{8}$ Besides, it is a practical and frequently preferred imaging method in the emergency setting. However, there are limited data regarding the appearance of WE lesions on $\mathrm{DWI}^{8}$ and it is unclear if DWI is sensitive enough to reveal WE lesions. ${ }^{9}$

\section{Conclusion}

In conclusion, via the presentation of this case, we remark on the importance of repeating neuroimaging in cases with clinical suspicion of WE, but with negative initial MRI results. We interrogate the rationale of MRI-based diagnosis in WE. Future studies of larger cases including the temporal evaluation of the MRI characteristics of WE are warranted to better understand the underlying mechanisms and increase the diagnostic accuracy.

\section{Conflict of Interest: None}

Source(s) of support: None

\section{References}

1. Butterworth RF, Gaudreau C, Vincelette J, Bourgault AM, Lamothe F, Nutini AM. Thiamine deficiency and Wernicke's encephalopathy in AIDS. Metab Brain Dis. Dec 1991;6(4):207-212. https://doi.org/ 10.1007/BF00996920

2. Isen DR, Kline LB. Neuro-ophthalmic Manifestations of Wernicke Encephalopathy. Eye Brain. 2020;12:4960. https://doi.org/10.2147/EB.S234078

3. Chaves LC, Faintuch J, Kahwage S, Alencar Fde A. A cluster of polyneuropathy and WernickeKorsakoff syndrome in a bariatric unit. Obes Surg. Jun 2002;12(3):328-334. https://doi.org/ 10.1381/096089202321088093

4. Zuccoli G, Santa Cruz D, Bertolini M, et al. MR imaging findings in 56 patients with Wernicke encephalopathy: nonalcoholics may differ from alcoholics. AJNR Am J Neuroradiol. Jan 2009;30(1):171-176. https://doi.org/ 10.3174/ajnr. A 1280

5. C. W. Die akute hämorrhagische polioencephalitis superior. Lehrbuch der Gehirnkrankheiten für Ärzte und Studier. 1881(II):229-242. https://doi.org/ 10.1093/alcalc/agm144

6. Zuccoli G, Gallucci M, Capellades J, et al. Wernicke encephalopathy: MR findings at clinical presentation in twenty-six alcoholic and nonalcoholic patients. AJNR Am J Neuroradiol. Aug 2007;28(7):13281331. https://doi.org/ 10.3174/ajnr.A0544

7. Harper CG, Giles M, Finlay-Jones R. Clinical signs in the Wernicke-Korsakoff complex: a retrospective analysis of 131 cases diagnosed at necropsy. J Neurol Neurosurg Psychiatry. Apr 1986;49(4):341-345. https://doi.org/ 10.1136/jnnp.49.4.341

8. Manzo G, De Gennaro A, Cozzolino A, Serino A, Fenza G, Manto A. MR imaging findings in alcoholic and nonalcoholic acute Wernicke's encephalopathy: a review. Biomed Res Int. 2014;2014:503596. https:// doi.org/ 10.1155/2014/503596

9. Zhong C, Jin L, Fei G. MR Imaging of nonalcoholic Wernicke encephalopathy: a follow-up study. AJNR Am J Neuroradiol. Oct 2005;26(9):2301-2305. 